\title{
Morphometric Assesment of the External Anatomy of Fourth Ventricle and Dorsal Brainstem in Fresh Cadavers
}

\author{
Veysel ANTAR ${ }^{1}$, Okan TURK ${ }^{1}$, Salim KATAR ${ }^{2}$, Mahmut OZDEN ${ }^{3}$, Balkan SAHIN ${ }^{4}$, Sahin YUCELI ${ }^{5}$, \\ Erdogan KARA ${ }^{6}$, Ayse YURTSEVEN ${ }^{6}$
}

\author{
${ }^{1}$ Istanbul Training and Research Hospital, Department of Neurosurgery, Istanbul, Turkey \\ ${ }^{2}$ Selahattin Eyyubi City Hospital, Department of Neurosurgery, Diyarbakir, Turkey \\ ${ }^{3}$ Bahcesehir University, Department of Neurosurgery, Istanbul, Turkey \\ ${ }^{4}$ Sultan Abdulhamit Han Training and Research Hospital, Department of Neurosurgery, Istanbul, Turkey \\ ${ }^{5}$ Erzincan Neon Hospital, Department of Neurosurgery, Erzincan, Turkey \\ ${ }^{6}$ Ministry of Justice, Council of Forensic Medicine, Istanbul, Turkey
}

Corresponding author: Veysel ANTAR docguard@hotmail.com

\section{ABSTRACT}

AIM: To investigate the external anatomy of the fourth ventricle and dorsal brainstem using morphometric data, which could be useful for preoperative surgical planning.

MATERIAL and METHODS: Between January 2017 and December 2017, 42 fresh adult cadavers were investigated for the measurements of the cadaver brainstems and fourth ventricle, and they were recorded by photography. Measurements were evaluated according to body mass indexes (BMls) of the patients. We also investigate the visualization of facial colliculus and stria medullaris on brainstem.

RESULTS: A total of 42 fresh cadavers with a mean age of $45.38 \pm 16.41$ years old were included in this research. We found no statistically significant difference between measurements and BMls. Facial colliculus was visualized in $92.9 \%$ ( $n=39$ ), but it could not visualized in $7.1 \%(n=3)$ of the subjects. When the right side of the stria medullaris was examined, one bundle was seen in $59.5 \%(n=25)$ of the subjects, two bundles were seen in $31 \%(n=13)$ of the subjects, and 3 bundles were seen in $9.5 \%(n=4)$ of the subjects. When the left side of the stria medullaris was examined, one bundle was seen in $57.1 \%(n=24)$ of the subjects, two bundles were seen in $33.3 \%(n=14)$ of the subjects, and three bundles were seen in $9.5 \%(n=4)$ of the subjects.

CONCLUSION: Knowledge of the normal morphometry of the dorsal brainstem as it has been reported in this study will help one to assess distortions in any preoperative imaging studies and surgical planning.

KEYWORDS: Fourth ventricle, Dorsal brainstem, Fresh cadaver, Morphometric study

\section{INTRODUCTION}

$\mathrm{T}$ The surgical management of fourth ventricle and brainstem pathologies, particularly with regard to tumors, remains a surgical challenge (8). Therefore, a detailed knowledge of the anatomy of the area involved, and a determination of the safe entry points are key factors involved in a successful surgical outcome (3).
In the literature, various surgical techniques and approaches have been developed previously, and morphometric studies have been conducted using either imaging methods or cadavers $(1,2,4,5,6,9,10,15)$. However, most of the cadaveric studies were conducted using formalin and alcohol-fixed cadavers, and they were comprised of small sample sizes $(1,2,15)$. Unfortunately, formaldehyde can cause structural changes in the brainstem, which can affect the morphometric values.
Veysel ANTAR : (1): 0000-0003-2144-6442

Okan TURK (D): 0000-0002-0074-2835

Salim KATAR (1): 0000-0002-8311-1122
Mahmut OZDEN (10): 0000-0003-2441-0015

Balkan SAHIN (D) : 0000-0003-4761-8871

Sahin YUCELI (D) : 0000-0002-9471-3575
Erdogan KARA

(1) : 0000-0002-5695-5709

Ayse YURTSEVEN (1) : 0000-0002-8889-789X 
We believed that a determination of the external anatomy of the dorsal brainstem and fourth ventricle using fresh cadavers could provide valuable morphometric data that was similar to the anatomy that neurosurgeons would observe while conducting surgery.

\section{MATERIALS and METHODS}

Forty-two fresh adult cadavers were investigated between January 2017 and December 2017. This study was conducted prospectively in cooperation with the Istanbul Training and Research Hospital Department of Neurosurgery and the Istanbul Forensic Medicine Institution, with approval of the Republic of Turkey Ministry of Justice Forensic Medicine Institution's Research Committee(B.03.1.ATK.0.01.00.08/705).

In this study, we performed our measurements by excluding any diseases that may have altered the size of the brain tissue (trauma-related parenchymal injuries, non-traumatic intracranial hemorrhages, ischemic systemic diseases, and intracranial tumors). Additionally, in order to avoid individual confounding the variables, these measurements were performed by two independent investigators, and then they were evaluated by a third independent investigator. The fourth ventricle measurements were assessed by obtaining the mean of the readings of the two investigators (Figure 1).

The measurements were made of the cadaver brainstems, and they were recorded by photography (Figure 2-4). Two calvarias were opened in those cases without histopathological evaluation sampling, and they were cut using tentorium scissors. The brainstem was removed from the foramen magnum, and then the whole brain was removed. The brainstem was separated and the measurements were

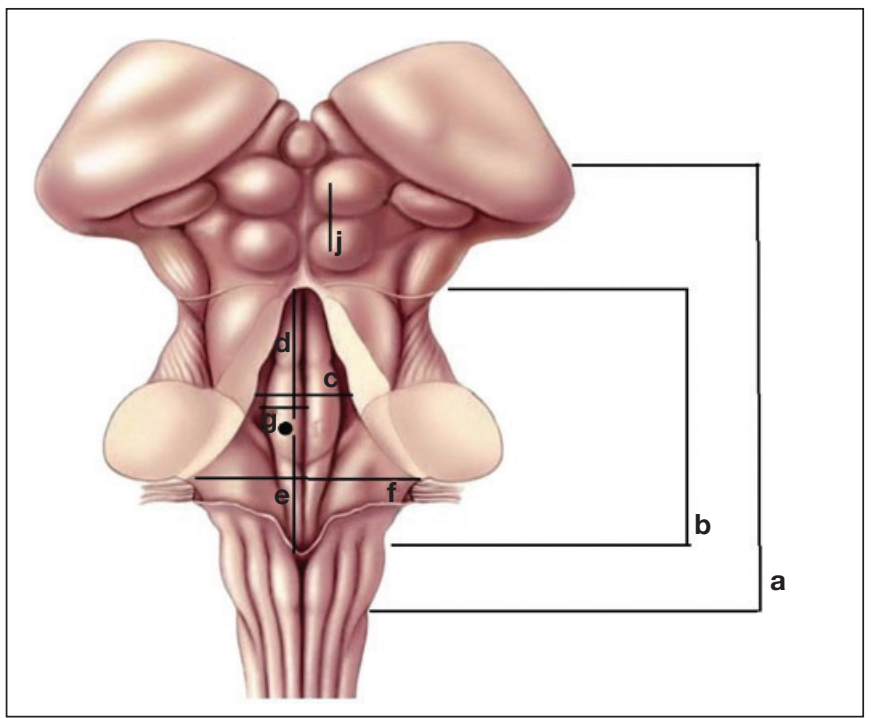

Figure 1: a: Length of the Brainstem, b: Aqueduct Obex (mm), c: Lateral Recess Length $(\mathrm{mm})$, d: Facial Colliculus - Obex, e: Aqueduct - Facial Colliculus, f: Foramen Luschka (Distance between right and left), g: Distance between Median Sulcus,Sulcus Limitans, j: Distance between the midlines of Superior and Inferior Colliculus. made. After a short flushing process, the brainstem was set after the separation and predetermined distances were measured using millimeter rulers. The heights and weights of the cadavers were measured, and the body mass indexes were calculated.

\section{Statistical Analysis}

The Number Cruncher Statistical System 2007 (NCSS, LLC., Kaysville, UT, USA) was used for the statistical analysis. The Student's t-test was used for the comparison of the descriptive statistical methods (mean, standard deviation, median,

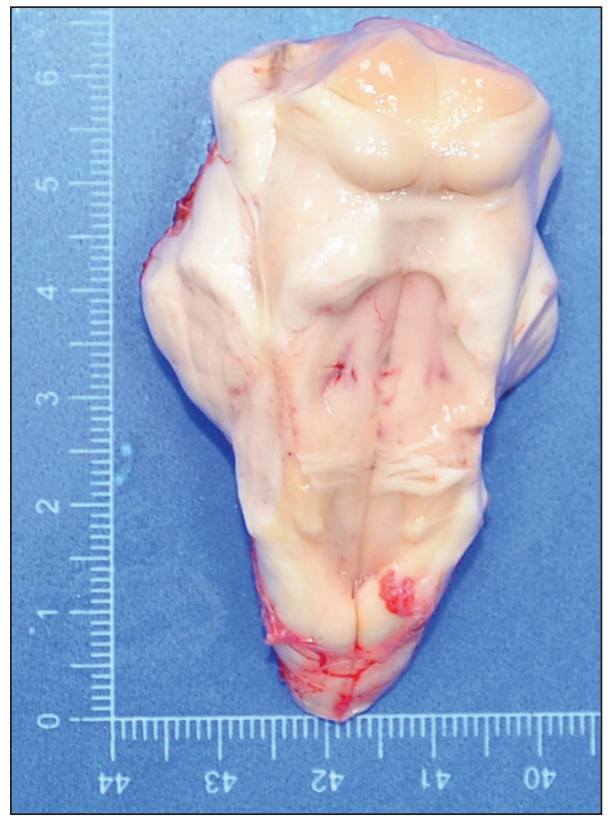

Figure 2: The photographic image of brainstem sampled from fresh cadaver.

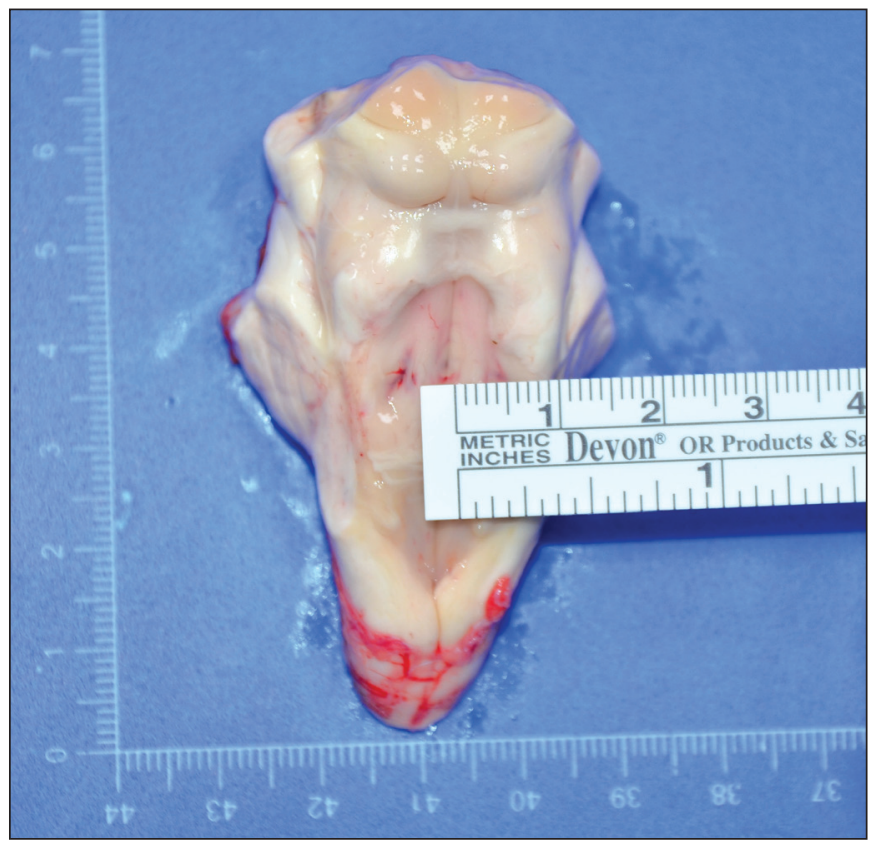

Figure 3: The sample image of brainstem and horizontal measurement technique. 
frequency, and ratio) as well as the normal distribution of the comparisons of the quantitative data between the groups. The Pearson's correlation analysis was used to evaluate the intervariable correlations. The results were evaluated at a confidence interval of $95 \%$ and a significance level of $p<0.05$.

\section{RESULTS}

A total of 42 fresh cadavers (26 males and 16 females) with a mean age of $45.38 \pm 16.41$ years old (min-max:16-76 years) were included in this research. The demographic features of the cadavers are shown in Table I. The brainstem measurements are shown in Table II.

The correlations between the ages, body mass indexes (BMls), and brain structures are shown in Table III. We found no statistically significant difference between measurements and BMls. There were no correlations between the brain structure features and the ages $(p>0.05)$; however, there were negative and weak correlations between the ages and the aqueduct obex, facial colliculus, and measurement from aqueduct to facial colliculus. In addition, there were positive and weak correlations between the brainstem lengths, aqueduct obex, pons, and measurement from median to sulcus limitans.

The correlation analyses between the gender and brain structure features are shown in Table IV. However, no significant differences were observed between the gender and the brain structures $(p>0.05)$.

When determining whether or not the facial colliculus was visualized, it could be visualized in $92.9 \%(n=39)$ of the subjects, but it could not be visualized in $7.1 \%(n=3)$ of the subjects. When the right side of the stria medullaris was examined, one bundle was seen in $59.5 \%(n=25)$ of the subjects, two bundles were seen in $31 \%(n=13)$ of the subjects, and 3 bundles were seen in $9.5 \%(n=4)$ of the subjects. When the left side of the stria medullaris was examined, one bundle was seen in $57.1 \%$ $(n=24)$ of the subjects, two bundles were seen in $33.3 \%(n=14)$ of the subjects, and three bundles were seen in $9.5 \%(n=4)$ of the subjects.

\section{DISCUSSION}

The knowledge of the external anatomy of the brainstem plays an important role in surgical planning, because of the correspondence between the external-internal structures and the anatomical landmarks on the brainstem. The morphology of the dorsal brainstem is also important in surgical approaches for the fourth ventricle tumors. The dorsal surface of the brainstem has an intraventricular portion, the rhomboid fossa, which is the floor of the fourth ventricle, and an extraventricular portion represented by the tectal plate and the cerebellar peduncles (14). The rhomboid fossa has the shape of a diamond and is delineated by the internal margins of the superior and inferior cerebellar peduncles. The lateral angles of the rhombus are closed by the middle cerebellar peduncles, the inferior angleis the obex, and the superior corner is marked by the frenulum of the superior medullary velum of the fourth ventricle (14).

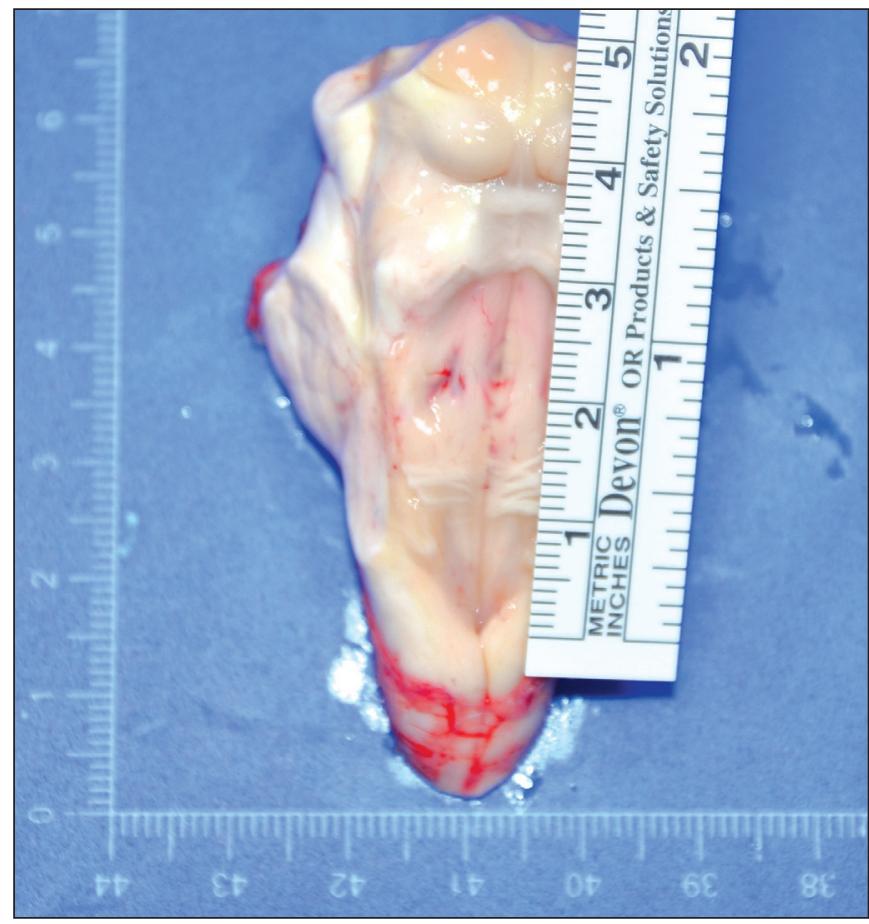

Figure 4: The sample image of brainstem and vertical measurement technique.

Table I: Demographical Features $(n=42)$

\begin{tabular}{|c|c|c|c|}
\hline \multicolumn{2}{|l|}{ Feature } & Min-Max & Mean \pm SD \\
\hline \multicolumn{2}{|c|}{ Age (year) } & $16-76$ & $45.38 \pm 16.41$ \\
\hline \multicolumn{2}{|c|}{ Height (cm) } & $145-185$ & $167.50 \pm 9.40$ \\
\hline \multicolumn{2}{|c|}{ Weight (kg) } & $52-145$ & $80.48 \pm 18.88$ \\
\hline \multirow{2}{*}{\multicolumn{2}{|c|}{ BMI }} & $18-48$ & $28.72 \pm 6.46$ \\
\hline & & $\mathbf{n}$ & $\%$ \\
\hline \multirow{2}{*}{ Gender } & Female & 16 & 38.1 \\
\hline & Male & 26 & 61.9 \\
\hline
\end{tabular}

With the recent advances in imaging technology, particularly in magnetic resonance imaging (MRI), it has become possible to achieve reliable and accurate neuroanatomical views (10). In parallel with recent developments, the number of neuroanatomical studies using MRI has also increased. Several studies have focused on the morphometric measurements in live persons or in postmortem research; however, they yielded conflicting results with regard to the effects of age or gender on the brain structures $(6,7,9,10,11,13)$.

Shah et al. assessed the posterior fossa structures using midsagittal MRI in which they highlighted the age-related decline in the cross-sectional area of the midbrain and the striking intercorrelations between the pons, medulla oblongata, and cerebellar vermis dimensions (13). In a 
prospective MRI study done by Raz et al., the age and sex differences in the cerebellum and the ventral pons were reported, and they concluded that the cerebellum and pons were larger in men. Luft et al. investigated the time course and regional differences in the age-related volume loss in the cerebellum and volume by using three-dimensional MRI in healthy volunteers $(7,11)$. However, in contrast with the aforementioned studies, they did not find any effects of the age on the brainstem volume. These conflicting results could be attributed to the regional factors, genetics, or different demographical features of the study populations. It should also be kept in mind that the reliability and exactness of MRI-based volumetry may change depending on the slice thickness, size, and form of the subject (12). The mean age of the study samples may also have affected the results of the studies. For example, volumetric differences may be seen in the later age periods of life (60s or $70 \mathrm{~s}$ ) due to atrophy of the cortex. Moreover, postmortem changes, such as atrophy, can be seen in the brains of cadavers, and it is not always possible to access certain parts of the brain in three-dimensional MRI of a living person. These challenges create drawbacks to the morphometric measurements. Therefore, in our study, we used fresh cadavers so that the brainstem structures were as similar to those of live persons as possible. Another important issue to be highlighted was the fact that this was the first study considering the morphometric measurements of the brainstem in a Turkish population. The mean lengths of the brainstem, medulla oblongata, pons, and cerebellum were $54.37 \mathrm{~mm}, 16.43 \mathrm{~mm}, 29.6 \mathrm{~mm}$, and $14.8 \mathrm{~mm}$, respectively. Negative and weak correlations were found between the ages and the aqueduct obex, facial colliculus, and aqueduct facial colliculus. In addition, there were positive and weak correlations between the brainstem lengths, aqueduct obex, pons, and median sulcus limitans. No significant differences were observed between the genders and the brain structures (p>0.05).

Szabo et al. performed a research on 10 formalin fixed brainstem specimens, which were harvested with respect to the topographic anatomy and their macroscopic measurements were performed with a Vernier caliper (14). They evaluated many anatomic structures but similarly to us they measured facial colliculi paramedially located at the base of the triangle

Table II: Morphometric Features of the Brainstem $(n=42)$

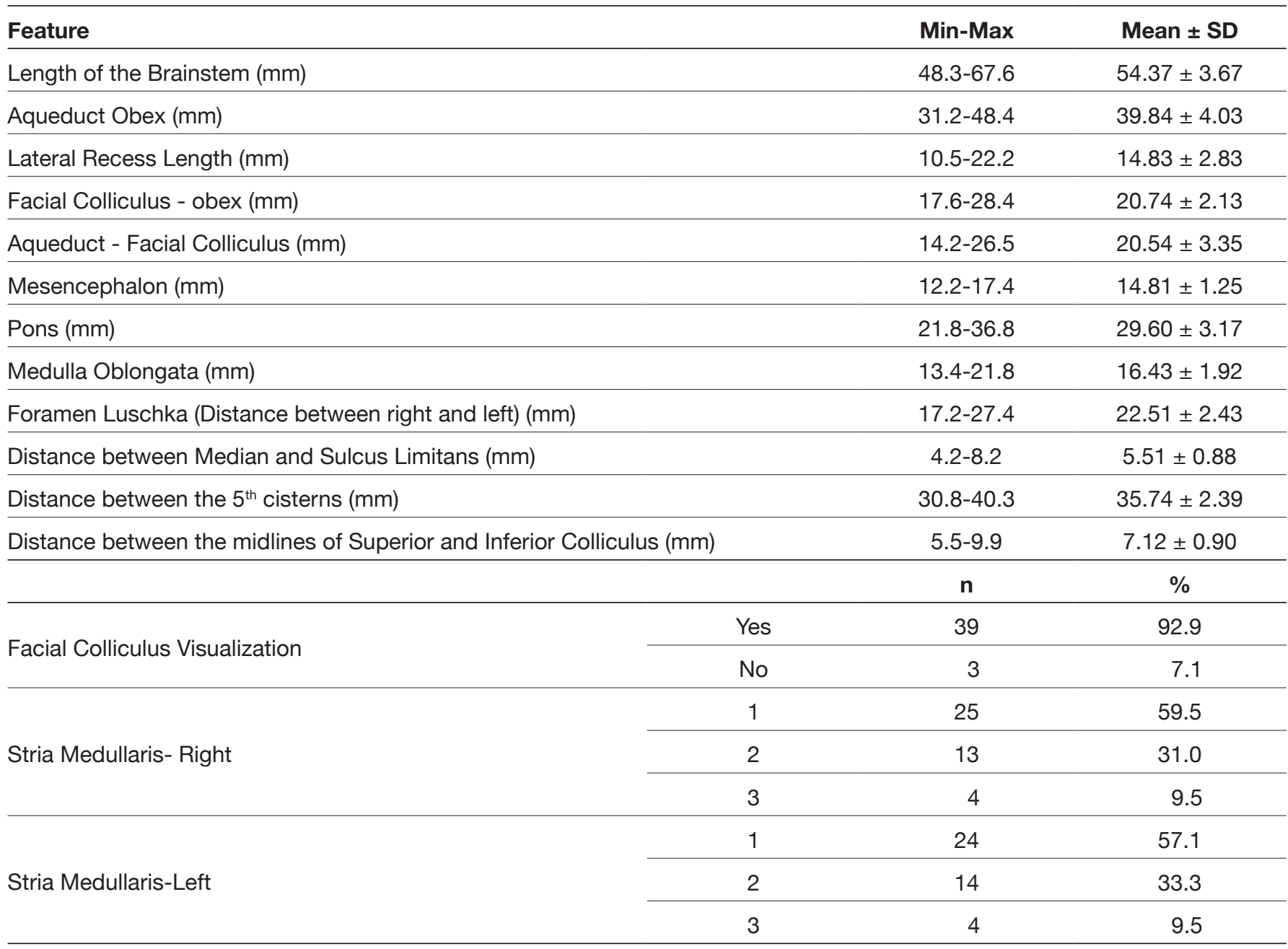


as $4.25 \mathrm{~mm}$ wide and continue superiorly with the $3.4 \mathrm{~mm}$ wide median eminences delineated by sulcus limitans. Our mean value for distance from medain to sulcus limitans is 5.51 $\mathrm{mm}$. As we thought our values are longer because formalin fixed brains could shrink and atrophied.

Rajaei et al. evaluated the dimensions of various parts of the pons, and they compared the data regarding the age and gender by using MRI (10). In addition, Muthukumar et al. reported the relationship between the morphometric analysis of the foramen magnum region and the transcondylar approach in dry skulls (9). Moreover, Yagmurlu et al. described the three-dimensional microsurgical anatomy and safe entry zones of the brainstem in 15 cadavers (15). Two studies taking into account the anatomical aspects of the surgical approach through the floor of the fourth ventricle were reported by Bogucki et al. $(1,2)$.

Table III: Correlations Between Age, Body Mass Index, and Brainstem Morphometry $(n=42)$

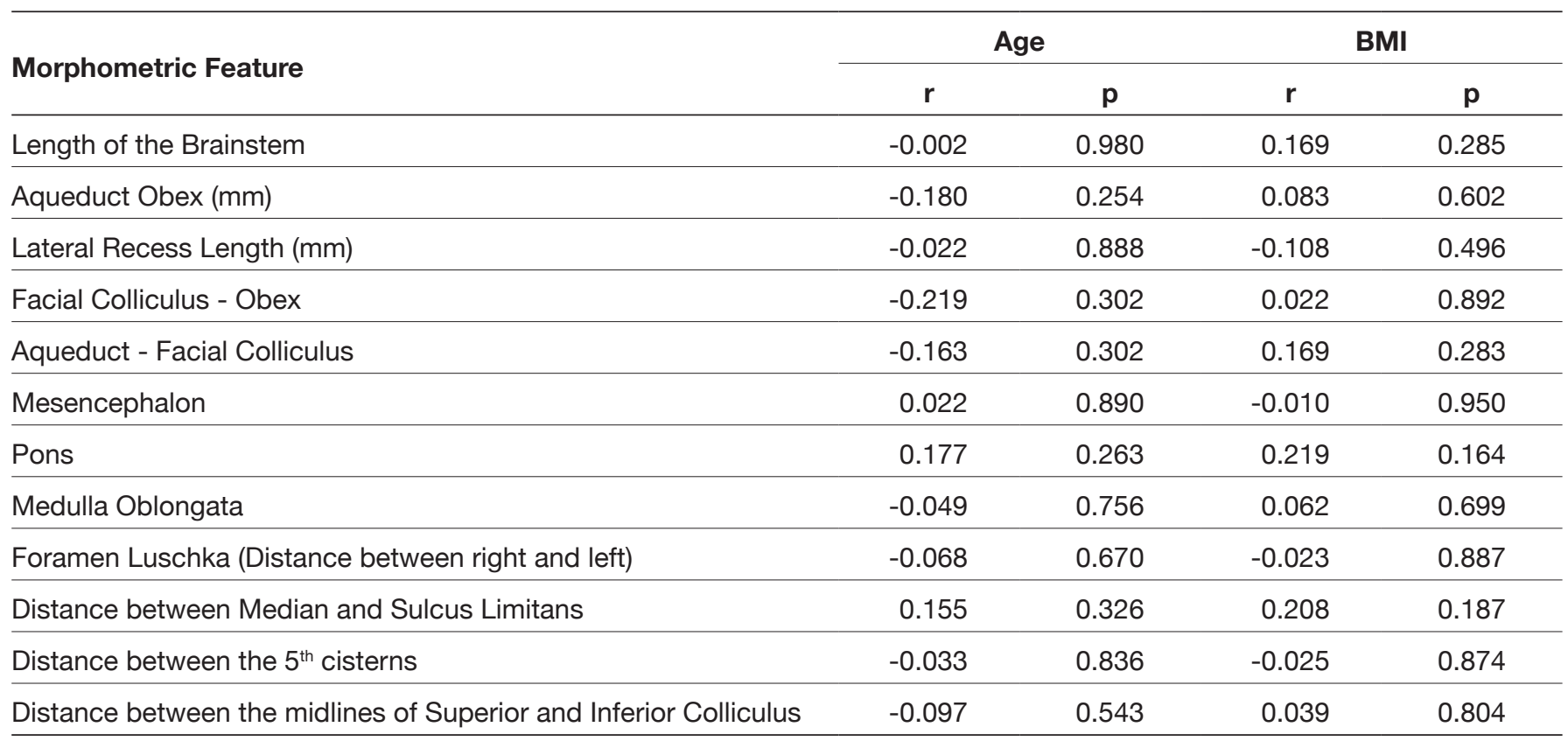

$r$ : Pearson's correlation coefficients.

Table IV: Correlation Between Gender and Brainstem Morphometry $(n=42)$

\begin{tabular}{|c|c|c|c|c|c|}
\hline \multirow{2}{*}{ Morphometric Feature } & \multicolumn{2}{|c|}{ Male } & \multicolumn{2}{|c|}{ Female } & \multirow{2}{*}{$\mathbf{p}^{*}$} \\
\hline & Mean & SD & Mean & SD & \\
\hline Length of the Brainstem & 54.50 & 4.29 & 54.16 & 2.47 & 0.928 \\
\hline Aqueduct Obex (mm) & 39.51 & 4.88 & 40.38 & 2.08 & 0.486 \\
\hline Lateral Recess Length (mm) & 15.39 & 3.06 & 13.92 & 2.21 & 0.195 \\
\hline Facial Colliculus - Obex & 20.74 & 2.31 & 20.74 & 1.86 & 0.866 \\
\hline Aqueduct - Facial Colliculus & 20.31 & 3.65 & 20.90 & 2.88 & 0.492 \\
\hline Mesencephalon & 14.67 & 1.30 & 15.05 & 1.16 & 0.585 \\
\hline Pons & 29.45 & 3.39 & 29.83 & 2.86 & 0.669 \\
\hline Medulla Oblongata & 16.51 & 1.86 & 16.28 & 2.07 & 0.492 \\
\hline Foramen Luschka (Distance between right and left) & 22.39 & 2.67 & 22.72 & 2.05 & 0.959 \\
\hline Distance between Median and Sulcus Limitans & 5.57 & 0.98 & 5.42 & 0.72 & 0.785 \\
\hline Distance between the $5^{\text {th }}$ cisterns & 35.82 & 2.64 & 35.61 & 1.98 & 0.746 \\
\hline Distance between the midlines of Superior and Inferior Colliculus & 7.31 & 0.91 & 6.80 & 0.82 & 0.052 \\
\hline
\end{tabular}

*Student's t-test 
In the macroscopic view of the base of the fourth ventricle, knowing the distance from the sulcus limitans, which separates the motor and sensorial nuclei, to the median sulcus can roughly provide the surgeon with information about the incision site. Therefore, this study aimed at helping to identify the safe entry zone sites in the surgical interventions of brain stems with disturbed anatomical structures due to tumors or associated conditions. Similarly, the measurements of safe zones with respect to landmarks may help surgeons to determine these regions during the perioperative period.

The facial colliculus is an important anatomical structure at the base of the fourth ventricle. The facial colliculus, formed by fibers from the motor nucleus of the facial nerve, is an elevated area located on the dorsal pons in the floor of the fourth ventricle. The facial colliculus is an important anatomical landmark because the nucleus of the sixth cranial nerve is located beneath it (12). The identification of the facial colliculus for fourth ventricle surgery, especially for performing surgical incisions both above and below this structure (two safe entry zones: i.e., suprafascicular and infrafascicular), is very critical. Therefore, the distances from the facial colliculus to both the obex and the aqueduct were measured. We believe that in such conditions in which the facial colliculus or sulcus limitans cannot be visualized, or when it is covered by a tumor mass, these measurements will provide information during the perioperative period that will greatly help the surgeon to perform the incision accordingly. In our study, we also checked whether or not the facial colliculus was visible in the fresh cadavers. According to our results, $92 \%$ of the facial colliculi were visible.

\section{CONCLUSION}

In light of our first and preliminary results, the brainstem morphometric measurements did not change according to the age, gender, and BMI in Turkish population. Knowledge of the normal morphometry of the dorsal brainstem as it has been reported in this study will help one to assess distortions in any preoperative imaging studies and surgical planning.

\section{- REFERENCES}

1. Bogucki J, Gielecki J, Czernicki Z: Digital-image analysis of the rhomboid fossa surface. Folia Morphol (Warsz) 56(3):129135, 1997

2. Bogucki J, Gielecki J, Czernicki Z: The anatomical aspects of a surgical approach through the floor of the fourth ventricle. Acta Neurochir (Wien) 139(11):1014-1019, 1997
3. Cavalcanti DD, Preul MC, Kalani MY, Spetzler RF: Microsurgical anatomy of safe entry zones to the brainstem. J Neurosurg 124(5):1359-1376, 2016

4. Gielecki J, Bogucki J, Czernicki Z: Digital-image analysis of the rhomboid fossa surface. Folia Morphol (Warsz) 55(4):240242, 1996

5. Koutourousiou M, Filho FV, Costacou T: Pontine encephalocele and abnormalities of the posterior fossa following transclival endoscopic endonasal surgery. J Neurosurg 121(2):359-366, 2014

6. Lee BY, Sohn JH, Choi MH, Lee SJ, Kim HS, Yang JW, Choi JS, Kim HS, Yi JH, Tack GR, Chung SC: A volumetric study of the corpus callosum in 20s and 40s Korean people. Brain Struct Funct 213(4-5):463-467, 2009

7. Luft AR, Skalej M, Welte D, Kolb R, Klose U: Reliability and exactness of MRI-based volumetry: A phantom study. J Magn Reson Imaging 6(4):700-704, 1996

8. Mehta VS, Chandra PS, Singh PK, Garg A, Rath GK: Surgical considerations for 'intrinsic' brainstem gliomas: Proposal of a modification in classification. Neurol India 57(3):274-281, 2009

9. Muthukumar N, Swaminathan R, Venkatesh G, Bhanumathy SP: A morphometric analysis of the foramen magnum region as it relates to the transcondylar approach. Acta Neurochir (Wien) 147(8):889-895, 2005

10. Rajaei F, Salahshoor MR, Hashemi HJ, Haghdoost-Yazdi H, Pahlevan AA: Morphometric study on dimensions of various parts of pons and comparison of data in accordance with age and sex of healthy people by MRI. Neurol Res 31(10):10751078, 2009

11. Raz N, Gunning-Dixon F, Head D, Williamson A, Acker JD: Age and sex differences in the cerebellum and the ventral pons: A prospective MR study of healthy adults. AJNR Am J Neuroradiol 22(6):1161-1167, 2001

12. Sala F, Manganotti P, Tramontano V, Bricolo A, Gerosa M: Monitoring of motor pathways during brain stem surgery: What we have achieved and what we still miss? Neurophysiol Clin 37(6):399-406, 2007

13. Shah SA, Doraiswamy PM, Husain MM, Figiel GS, Boyko OB, McDonald WM, Ellinwood EH Jr, Krishnan KR: Assessment of posterior fossa structures with midsagittal MRI: The effects of age. Neurobiol Aging 12(4):371-374, 1991

14. Szabo BA, Pascalau R, Padurean VA: Morphometric study of the human brainstem and its neurovascular relations. Turk Neurosurg 28(5):768-775, 2018

15. Yagmurlu K, Rhoton AL Jr, Tanriover N, Bennett JA: Threedimensional microsurgical anatomy and the safe entry zones of the brainstem. Neurosurgery 10(4):602-619, 2014 